\title{
Experimental Studies and Modeling of Acoustic Instabilities in a Gas Turbine Model Combustor
}

\author{
YunTao Chen ${ }^{*}$ and James F. Driscoll ${ }^{\dagger}$ \\ Department of Aerospace Engineering, University of Michigan, Ann Arbor, MI 48109, USA
}

Partially premixed combustion has the merits of lower $\mathrm{NO}_{\mathrm{x}}$ emission as well as higher efficiency. However practical applications of such technology have been hindered by acoustic instabilities generated in combustion chambers when gas turbine engines are operated in premixed mode. A thorough understanding of the physical processes which trigger and sustain this instability needs to be gained to aid the design of next generation low-emission high-efficiency gas turbine engines. In this work, acoustic instabilities manifested in the Gas Turbine Model Combustor (GTMC), which was developed at DLR Stuttgart by W. Meier and colleagues, were investigated. Specifically, the GTMC was operated with dimethyl ether (DME) in a fuel rich condition. Multi-point pressure measurements were carried out to characterize the dominant instability mode of the combustion chamber. Simultaneous Planar Laser-Induced Fluorescence (PLIF) of formaldehyde $\left(\mathrm{CH}_{2} \mathrm{O}\right)$ and pressure measurements were then made at a sustained frequency of $4 \mathrm{kHz}$. Flame surface densities, calculated from the flame edges detected in the PLIF images, were used as the indicator of flame heat release rate and determined both temporally and spatially. Finally a reduced order model was proposed to describe the observed combustion instability. Key predictions made by this model, such as instability frequency and pressure phase differences, agreed with experimental observations. Future work will focus on expanding present model to explore the effects of varying parameters on the combustion instability.

\section{Nomenclature}

$\begin{array}{ll}\Delta h_{\mathrm{g}}^{\circ} & \text { Lower heating value } \\ \dot{m} & \text { Mass flow rate } \\ \dot{Q} & \text { Total heat release rate } \\ \gamma & \text { Ratio of heat capacities } \\ \jmath & \text { Square root of -1 } \\ \omega & \text { System instability frequency } \\ \omega_{0} & \text { Plenum Helmholtz frequency } \\ \omega_{2} & \text { Combustion chamber Helmholtz frequency } \\ \phi & \text { Equivalence ratio } \\ \psi_{20} & \text { Combust. chamber/plenum phase difference } \\ \rho & \text { Density of mixture } \\ \rho_{\mathrm{fs}} & \text { Flame surface density } \\ \tau_{\mathrm{c}} & \text { Convection time scale } \\ \mathrm{St} & \text { Strouhal number } \\ \zeta & \text { Damping ratio } \\ A F R_{\mathrm{st}} & \text { Stoichiometric air-fuel ratio } \\ C & \text { Speed of sound } \\ D & \text { Element equivalent diameter } \\ l & \text { Element length } \\ l_{\mathrm{fs}} & \text { Flame surface length } \\ \end{array}$

$\begin{array}{ll}P & \text { Pressure } \\ S & \text { Element cross section area } \\ T & \text { Temperature } \\ t & \text { Time } \\ U & \text { Axial velocity } \\ V & \text { Element volume }\end{array}$

\section{Subscripts}

$0 \quad$ Plenum properties

1 Injector properties

2 Combustion chamber properties

3 Chimney properties

b Burnt mixture

r Real part (of a complex number)

$\mathrm{u} \quad$ Unburnt mixture

${ }^{*}$ Ph.D. candidate and research assistant, yuntaoc@umich.edu, Student Member.

${ }^{\dagger}$ Professor, jamesfd@umich.edu, Fellow. 


\section{Introduction}

Combustion instability is the major obstacle that needs to be overcome before premixed and partially

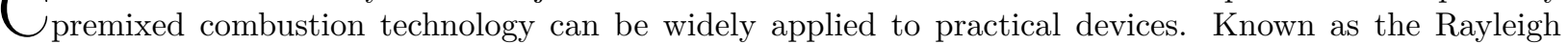
criteria, ${ }^{1}$ acoustic fluctuation draws power from the heat released by the flame and is thus amplified when pressure and heat release rate fluctuation are in phase. However, the factors that determine whether Rayleigh criteria is satisfied in a geometrically complicated swirl-combustor have yet been well understood. Experimental diagnostics as well as conceptual models are needed to fully comprehend such problem, which are the major goals of this work.

The Gas Turbine Model Combustor (GTMC) is the platform that the present study is based on. It was developed by W. Meier and colleagues at the German Aerospace Center (DLR) in Stuttgart. ${ }^{2}$ It has the advantage of a canonical axisymmetric swirler-design, yet still exhibiting the fundamental physics associated with flames in gas turbine engine combustors. It contains two swirling air streams, which surround an annular fuel stream, in a plenum-injector-combustor three component setup. Comprehensive measurements have been conducted at DLR Stuttgart by Meier and colleagues, including species and velocity fields, ${ }^{2,3}$ flame-flow interactions, ${ }^{4}$ precessing vortex core structures ${ }^{5}$ vortex-flame interactions,${ }^{6}$ and thermal-acoustic coupling. ${ }^{7,8,9}$ These measurements yield an elaborate database in terms of flow structures and flame characteristics for varying operating conditions, which makes this setup well calibrated for additional investigations. One key feature of the GTMC is that under certain fueling conditions, a self-sustained combustion instability would be established in the system.

Since the GTMC is both geometrically and physically complicated, the detailed modeling of such burner device can be very challenging. See and Ihme ${ }^{10}$ demonstrated that satisfactory simulation results could be achieved for certain operating conditions of the GTMC. However, the acoustically unstable cases have yet been tackled. As well, these high-fidelity simulations require extensive mesh-generation expertise and a large amount of computational power. It is hence attractive to develop a reduced order model that can capture the main physical processes in the combustor without too many computational complications.

Such approaches have been taken by several research groups on various experimental geometries. Notably, Hathout et al. ${ }^{11}$ studied the case where a combustion chamber with an inlet and an outlet acts as a Helmholtz resonator. Heat release rate of the combustion chamber was related to flame surface area, which was determined by local flame speed and inlet velocity, with the latter being the deciding factor. Pressure and velocity were correlated in the inlet pipe through conservation of momentum. In this way a second-order ordinary differential equation was then derived for the pressure fluctuations. Through stability analysis of the equation, the stable operating envelop was determined.

Researchers from Laboratoire EM2C at École Centrale Paris studied another set of setups. ${ }^{12,13}$ Specifically, Schuller et al. ${ }^{14}$ studied the acoustic coupling effects of a plenum-injector-combustor system using a reduced order model through matrix eigenvalue analysis. Palies et al. ${ }^{15}$ studied the same setup with fluctuating heat release rate, using the Flame Describing Function (FDF) framework. Furthermore, this analysis was compared to a Helmholtz solver by Silva et al., ${ }^{16}$ with good agreements.

Despite the large amount of high quality work related to this topic that have been done, there are still aspects of the GTMC that warrant attention. Firstly, both Steinberg et al. ${ }^{8,17}$ and Allison et al. ${ }^{9}$ have made extensive investigations on the thermal acoustic instabilities shown in the GTMC, and most evidences have indicated that the combustion chamber exhibits a Helmholtz mode. However, a detailed characterization of the combustion chamber instability mode has yet been conducted, which is necessary for the selection of an appropriate reduced order model. Secondly, all aforementioned reduced order models have specific assumptions and certain geometry dependence. A reduced order model tailored to the GTMC has yet been proposed. Thirdly, previous efforts have been made to conduct kilo-hertz simultaneous laser diagnostics with pressure measurements to characterize the thermal-acoustic instabilities of GTMC at the University of Michigan. ${ }^{18}$ Continuous improvements have since been made and their effects remain to be examined.

Therefore, the scope of the current work is to: $i$ ) characterize the dominant mode of instability in the combustion chamber of the GTMC, $i i$ ) examine the pressure-heat release interactions with the improved kilohertz simultaneous laser diagnostic setup, and iii) develop a reduced order model for combustion instability and access its applicability to the GTMC.

The structure of the paper is laid as such: firstly the experimental setup is introduced, then the results from our experimental measurements are presented and discussed. This is followed by the comparison of the predictions from the proposed reduced order model with our experimental observations. Lastly conclusions are drawn and the paper closes with acknowledgements. 


\section{Experimental Setup}

In this section, basic information regarding the GTMC, the details of our laser diagnostics setup, and pressure measurement procedures are discussed.

\section{A. Burner schematics and operating conditions}

A schematic drawing of the gas turbine model combustor (GTMC) is shown in figure 1. Details of the dimensions of this burner can be found in references from DLR publications. ${ }^{2}$ Here we briefly provide some of the essential parameters. The injector of the GTMC consists of a central air nozzle, an annular fuel nozzle, and a co-annular air nozzle. Both air nozzles supply swirling dry air at atmospheric pressure and temperature from a common plenum. The inner air nozzle has an outer diameter of $15 \mathrm{~mm}$ and the annular nozzle has an inner diameter of $17 \mathrm{~mm}$ and an outer diameter of $25 \mathrm{~mm}$. The measured swirl number is approximately 0.55 . Non-swirling fuel is provided through three exterior ports fed through the annular nozzle which is subdivided into 72 channels of dimension 0.5 $\mathrm{mm} \times 0.5 \mathrm{~mm}$. The width of the fuel annulus is less than 0.5 $\mathrm{mm}$. The exit plane of the central air nozzle and fuel nozzle lies $4.5 \mathrm{~mm}$ below the exit plane of the outer air annulus. The exit plane of the outer air annulus will be referred to as the burner surface. The combustion chamber has a square cross section of $85 \mathrm{~mm}$ in width and $110 \mathrm{~mm}$ in height. The exit of the burner has a tapered lid which leads to an exhaust chimney with a diameter of $40 \mathrm{~mm}$ and a height of $50 \mathrm{~mm}$. The burner is operated with fused silica windows, with a thickness of $1.5 \mathrm{~mm}$, for flame visualization. The burner was fired with dimethyl ether (DME) fuel. An external cylindrical chamber was used for the equal division of the fuel flow into three separate lines which lead to the fuel ports on the burner. Mass flow rates for air and fuel lines were controlled by sonically choked

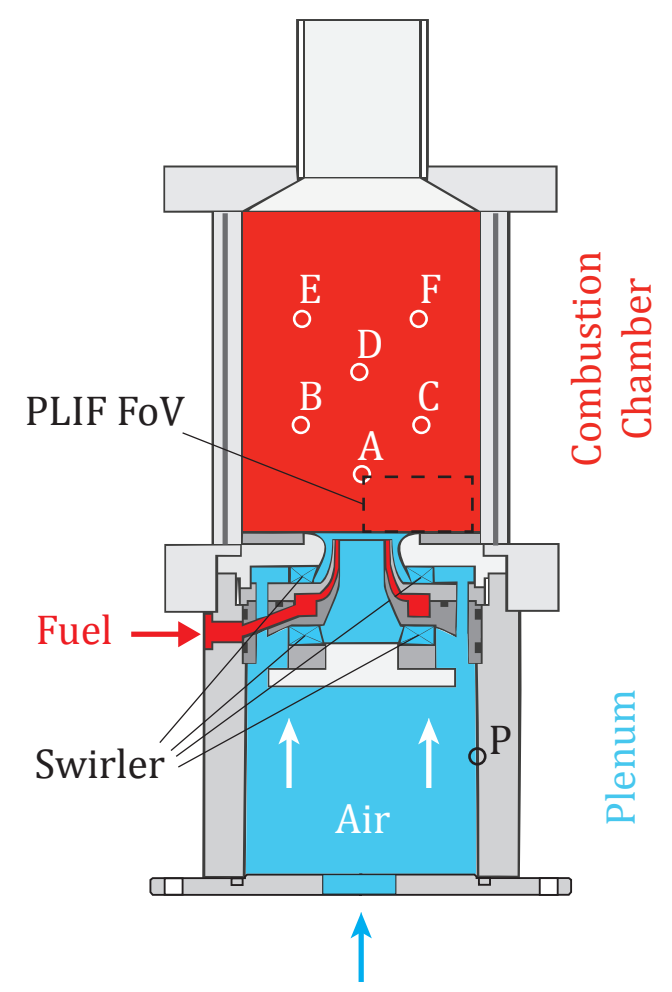

Figure 1. Schematic drawing of the GTMC ${ }^{2}$ orifices. In this study, the air flow rate was set to $282 \mathrm{~g} / \mathrm{min}$, which corresponds to the case B flame in the work of Weigand et al. ${ }^{2}$ The fuel flow rate was set to $38 \mathrm{~g} / \mathrm{min}$, resulting in an equivalence ratio of 1.2 .

\section{B. Multi-point pressure measurements}

To perform multi-point pressure measurements of the combustion chamber, one of the glass walls of the combustion chamber was replaced with a $9.5 \mathrm{~mm}$ thick steel plate. Six pressure taps were drilled into the wall so that pressure transducers could be mounted. Figure 1 shows the location of the these pressure ports in the combustion chamber (point "A" through "F"). The locations of these points on the combustion chamber wall are shown in table 1 . Here the symmetric axis of the burner is taken as $\mathrm{x}=0$ and the burner surface is taken as $\mathrm{y}=0$.

Table 1. Pressure measurement point locations*.

\begin{tabular}{lrrrrrr}
\hline Point & A & B & C & D & E & F \\
\hline \hline $\mathrm{x}[\mathrm{mm}]$ & 0 & -19 & 19 & 0 & -19 & 19 \\
$\mathrm{y}[\mathrm{mm}]$ & 19 & 38 & 38 & 57 & 76 & 76 \\
\hline${ }^{*} \mathrm{x}=0$ at burner symmetric axis and $\mathrm{y}=0$ at burner surface.
\end{tabular}

An additional pressure port was drilled onto the plenum wall and labeled point "P", also shown in figure 1. 
Two PCB piezoelectric pressure transducers were used to make the measurements. Returned signals were passed on to an oscilloscope (LeCroy Waverunner 6100A) for digital recording. The sampling rate of the oscilloscope was set to 25,000 samples per second and 5 seconds of data were recorded during each run. To eliminate hardware errors, one pressure transducer was fixed at point "B" while the other transducer rotated its location on all other pressure ports. Further calibration was provided by switching the two pressure transducers while measuring the phase differences between point "B" and "C". Pressure traces were recorded at a fixed burner surface temperature of $120^{\circ} \mathrm{F}$.

\section{Simultaneous pressure and laser diagnostics}

High-speed formaldehyde PLIF measurement was achieved with a frequency-tripled diode-pumped Nd:YAG Quantronix Hawk HP laser at $355 \mathrm{~nm}$. The laser was operated at $4 \mathrm{kHz}$ which produced pulse energies of $12 \mathrm{~mJ}$. The pulse length at this condition was about $120 \mathrm{~ns}$. The laser beams were formed into sheets and focused using cylindrical lenses. At the test section, the laser sheet had a height of $20 \mathrm{~mm}$. The formaldehyde fluorescence was observed using a combination of a LaVision HS-IRO intensifier and a Phantom v711 high-speed camera. A GG-385 filter was used to remove laser light interference. The intensifier and camera were operated at $4 \mathrm{kHz}$. At this rate the frame size of the camera was 1280 pixels $\times 800$ pixels. The region of interest in this study was $40 \mathrm{~mm} \times 20 \mathrm{~mm}$, covered by 1016 pixels $\times 508$ pixels. Hence the resolution of the image was $39 \mu \mathrm{m}$ per pixel. The resolving power of the optics was tested with a standard USAF target. Results showed that the system could resolve Group 2, element 5 to 6 on the target, which correspond to a resolving power of $7 \mathrm{lpmm}$ (line thickness $70 \mu \mathrm{m}$ ) at the laser sheet. During simultaneous measurements, the pressure data was retrieved from point "P" on the plenum, where a PCB pre-polarized ICP microphone $(378 \mathrm{C} 10)$ was mounted.

\section{Results and Discussions}

In this section, the results of our multi-point pressure measurements are presented, along with their implications. Then the data from our laser diagnostics are discussed.

\section{A. Characterization of combustion chamber instability mode}

The major acoustic mode of the GTMC has been subjected to several discussion before. Steinberg et al. ${ }^{8}$ postulated that the burner follows a multi-chamber Helmholtz mode. In their case it was demonstrated that 3 pressure ports drilled into the post of the combustion chamber of GTMC returned similar temporal pressure traces, with identical frequency peaks in power spectrum. Furthermore, they mentioned that pressure in the plenum is fluctuating at the same frequency, but with a phase lag of 60 to 80 degrees. Based on the trend of changes in instability frequencies, Allison et al. ${ }^{19}$ stated that it is more likely that the GTMC exhibits a combination of multiple modes. Since it is crucial in the process of understanding the combustion instability in the GTMC, the characterization of the instability mode in the combustion chamber had to precede all other measurements.

Table 2. Possible modes in the combustion chamber and their characteristics

\begin{tabular}{lll}
\hline Mode & Key parameter & Characteristics \\
\hline \hline Helmholtz & Phase differences & Pressure in phase across the volume \\
Standing wave & Pressure amplitude & Pressure amplitude varies from max to 0 \\
Vortex shedding & Strouhal number $(\mathrm{St})$ & Instability frequency controlled by St \\
\hline
\end{tabular}

By far the most widely accepted mode of instability in this combustor is of Helmholtz type. However standing waves have been observed previously in combustion systems with large aspect ratios. ${ }^{20}$ And it was shown that there are inner and outer recirculation zones generated by the injector in the combustion chamber of the GTMC. ${ }^{2}$ These recirculation zones are prone to vortex shedding, which can also generate instabilities. Each of these modes has its own characteristics, which are summarized in table 2. 


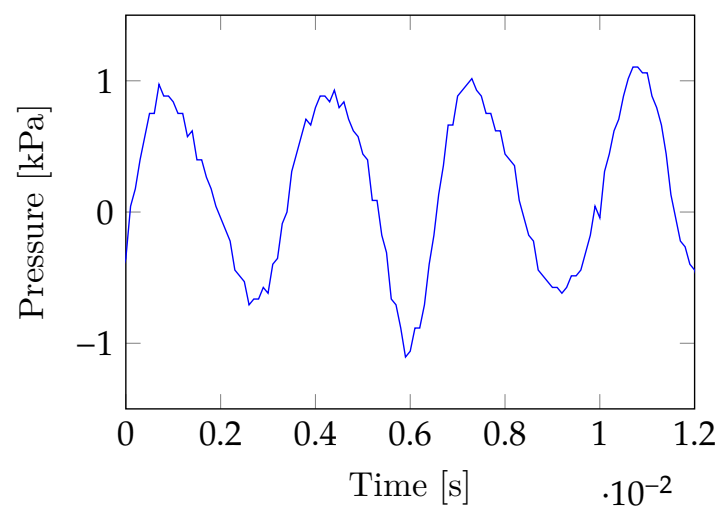

(a) Temporal pressure trace

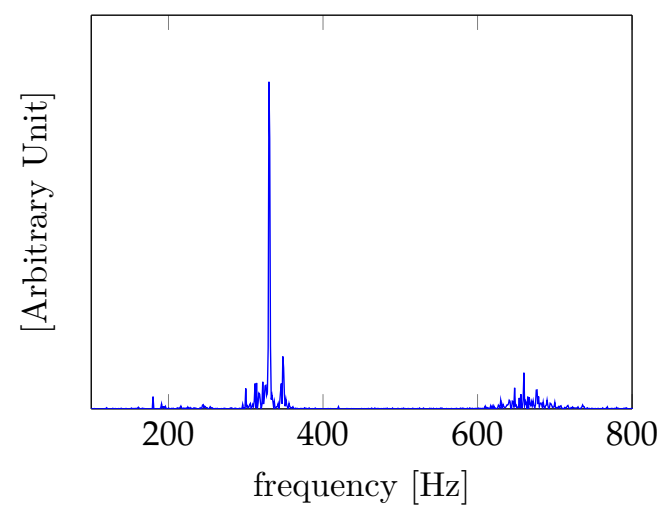

(b) Pressure Power Spectral Density

Figure 2. A typical pressure measurement result in combustion chamber

In present study, it is found that pressure signals returned from two pressure ports always fluctuate at the same frequency, with a very sharp peak in the frequency spectrum (as shown in figure 2(b)). At the current operating condition, the instability frequency was determined to be $330 \pm 2 \mathrm{~Hz}$. If vortex shedding caused instability inside the chamber, then its frequency would be determined by Strouhal number:

$$
\text { St }=\frac{2 \pi f \cdot L}{u},
$$

where $f, L$, and $u$ are characteristic frequency, length, and velocity respectively. If we choose $L$ to be $9 \mathrm{~cm}$ (burner surface edge length), $u$ to be $10 \mathrm{~m} / \mathrm{s}$ (average axial velocity ${ }^{2}$ ), and $\mathrm{St}$ to be $0.2,{ }^{21}$ then we could estimate the vortex shedding frequency $\left(f_{\mathrm{vtx}}\right)$ to be:

$$
f_{\mathrm{vtx}}=\frac{\mathrm{St} \cdot u}{2 \pi L} \approx 4 \mathrm{~Hz} .
$$

The result is two orders of magnitude smaller than what we observed in the experiment. Even though this is a very rough estimate, the discrepancy can not be explained by merely the error of estimation. This leads us to the conclusion that the observed instability at $330 \mathrm{~Hz}$ was not caused by vortex shedding.

Table 3. Relative phase between each measurement point and point $\mathbf{B}$, based on $\omega=330 \mathrm{~Hz}$

\begin{tabular}{ccccccc}
\hline & $\mathrm{P}$ & $\mathrm{A}$ & $\mathrm{C}$ & $\mathrm{D}$ & $\mathrm{E}$ & $\mathrm{F}$ \\
\hline \hline Phase difference & $50^{\circ} \pm 5^{\circ}$ & $-10^{\circ} \pm 5^{\circ}$ & $0^{\circ} \pm 5^{\circ}$ & $0^{\circ} \pm 5^{\circ}$ & $0^{\circ} \pm 5^{\circ}$ & $0^{\circ} \pm 5^{\circ}$ \\
w.r.t. B $\left(\psi_{B}-\psi_{*}\right)$ & & & & &
\end{tabular}

Relative phase differences between each measurement point and point "B" were calculated by Fourier transformation and shown in table 3. It shows that almost all the pressure ports inside the combustion chamber were in phase with each other. And the pressure inside the plenum was lagging for $0.44 \mathrm{~ms}$. Similar behaviors have also been observed by Steinberg et al. ${ }^{8}$ This demonstrates that the combustion chamber behaves like a Helmholtz resonator, where the pressure is in phase across the volume.

Furthermore, the correlation factors between each point and point "B" are shown in figure 3. As mentioned above, the frequency spectrum of pressure measurements shows a very sharp peak at the instability frequency. Which indicates that the pressure temporal trace is close to a single frequency sinusoid wave. For two of such waves, the correlation factor is given by $\cos (\Delta \psi)$, where $\Delta \psi$ is the relative phase difference between the two waves. In our case, since the phase differences between " $\mathrm{B}$ " and all other points in combustion chamber are close to zero, we would expect a near unity correlation factor. What figure 3 tells us instead, is that there are still irregularities within the time window during which we took the data. This has resulted in a correlation factor that is slightly lower than unity (about 0.8). Figure 3 nonetheless shows again that 
pressure inside the combustion chamber is strongly correlated across the volume, and the correlation with plenum is relatively weaker.

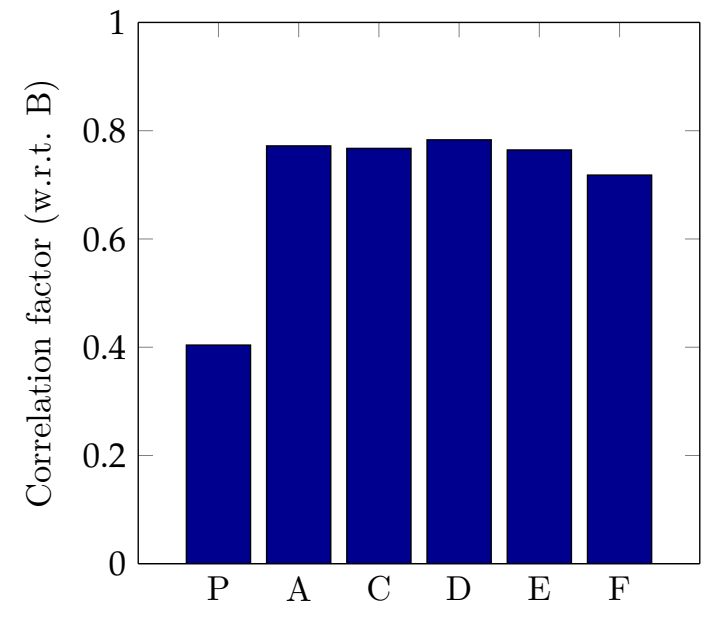

Figure 3. Correlation factors between each measurement point and point $B$

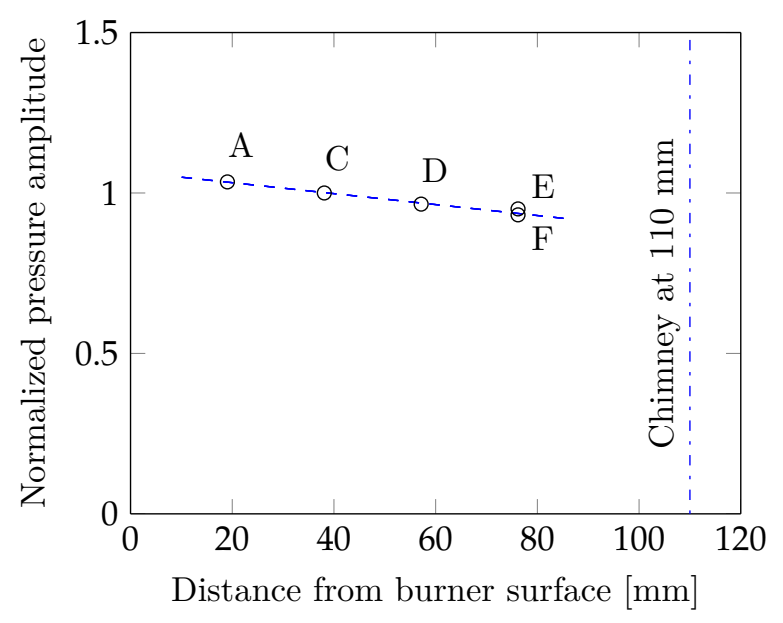

Figure 4. Normalized Pressure Amplitude

To check whether a standing wave is present in the combustion chamber, the pressure oscillation amplitudes at each point inside the combustion chamber are plotted in figure 4. If a longitudinal standing wave is present in the combustion chamber, we would see the pressure oscillation amplitudes fluctuate from maximum to zero at least once across the combustion chamber height. And near the chamber exit (chimney), the pressure oscillation should be close to zero. However the results shown in figure 4 contradict this assumption. We see that even though the pressure amplitudes decrease slightly as the distance from the burner surface increases, the change is relatively small (less than 10\%). Also, if we follow this linear extrapolation, the pressure oscillation at the chimney is far from zero. Hence the presence of a standing wave in the combustion chamber is also very unlikely. On the other hand, this figure does show that the presence of the large diameter chimney has some effects on the pressure oscillation amplitudes. The damping effect is more evident as we get closer to the chimney.

From the aforementioned investigations, we conclude that the dominant instability mode of the combustion chamber is indeed a Helmholtz mode. However, experimental data also shows that the instability mechanism deviates from a perfect Helmholtz mode, caused by flow features, geometry constraints, as well as heat release and damping effects.

\section{B. Flame surface measurements}

Allison et al. have demonstrated that high speed formaldehyde PLIF is a viable way to designate flame surfaces and hence determine the local heat release rate. ${ }^{19,22}$ It is considered to be an improvement over the conventional chemiluminescence detection, because the latter one is a line of sight measurement. The PLIF setup used in present study is based on the aforementioned study, but with improvements. Firstly, the field of view (as shown in figure 1) has been reduced to half of the burner surface to improve the resolving power and resolution of the optics (to $7 \mathrm{lpmm}$ and $39 \mu \mathrm{m}$ per pixel respectively). Additionally, an improved method for edge detection has also been developed. The previous method used local gradients to determine whether an edge in $\mathrm{CH}_{2} \mathrm{O}$ PLIF image is flame front or the boundary of thermal decomposition. The gradient calculation stencil in the previous study was about $300 \mu \mathrm{m}$ with a central differencing algorithm. With the improved resolution and resolving power, such method no longer fits present need. Calculating local gradient using a stencil size close to the resolving power of the optics will make it difficult to differentiate the two kind of edges. Hence an enlarged stencil based on the flame structure length scale was used. The central differencing algorithm has also been replaced by a single-maximum algorithm to better capture flame surfaces with large curvatures. A sample frame of the improved edge detection algorithm is shown in figure 5. It is shown that the new algorithm is capable of neglecting most of the edges that have visibly small gradients.

We assume that the integral of the flame surface area within each frame is proportional to the total heat 


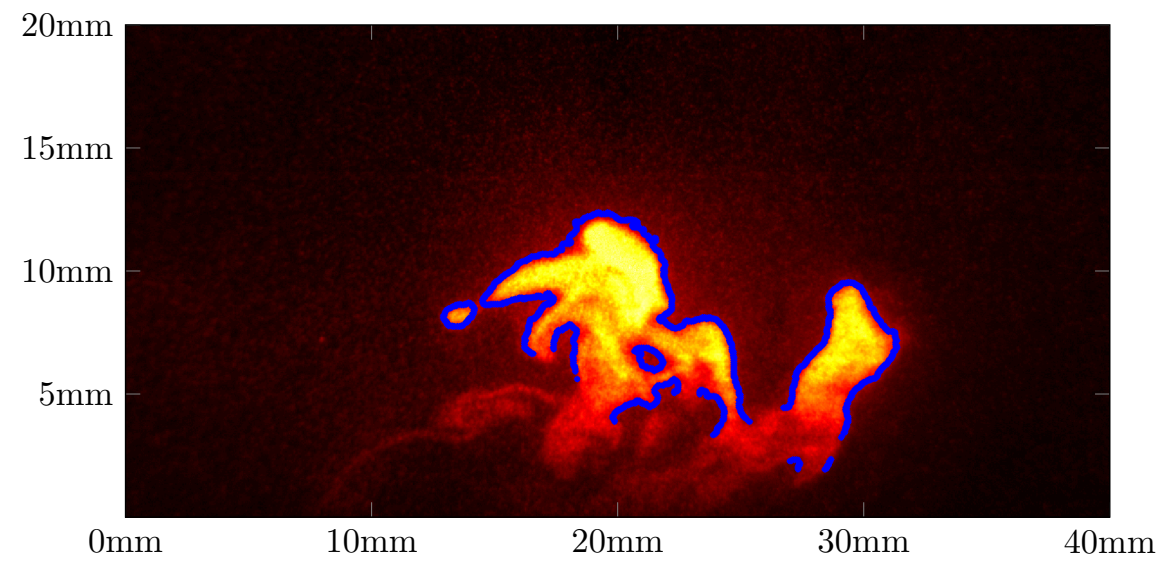

Figure 5. Sample image of $\mathrm{CH}_{2} \mathrm{O}$ PLIF data, with blue lines showing identified flame surface

release rate ( $A$ denotes the area of the entire frame):

$$
\dot{Q} \sim \sum_{A} l_{\mathrm{fs}},
$$

hence heat release rate fluctuations can be calculated from the fluctuations of the total flame surface area in each frame. Figure 6 compares the power spectral density of pressure oscillation and flame surface area oscillation.

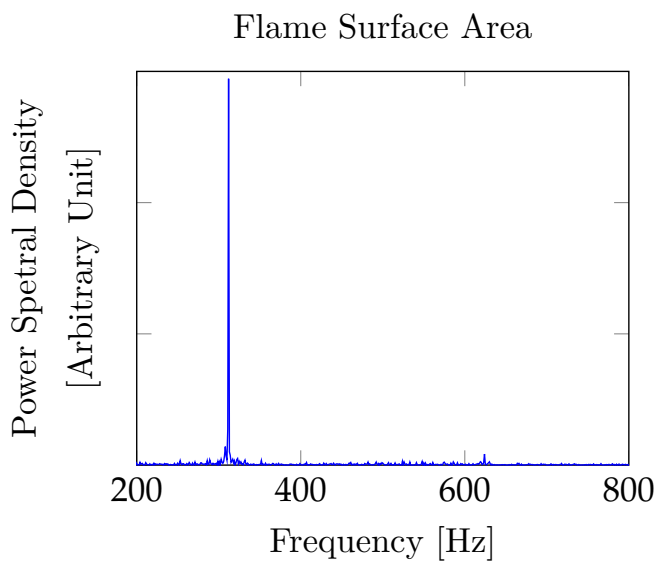

Combustion chamber pressure

Figure 6. Power spectrum of flame surface area and pressure

Similar to what has been observed previously, ${ }^{8,19}$ we can see that the flame surface area, and in this way heat release rate, is fluctuating almost at the the same frequency as the combustion chamber pressure. The peak of flame surface area spectrum in figure 6 is at $313 \mathrm{~Hz}$ and the peak of pressure spectrum is at 314 $\mathrm{Hz}$. Because the resolution of our FFT data processing routine is $1 \mathrm{~Hz}$, the difference in peak frequencies in figure 6 is within uncertainty limit. We also notice that the measured instability frequency here is 314 $\mathrm{Hz}$, whereas in the multi-point pressure measurement (section A) the instability frequency was $330 \mathrm{~Hz}$. In section A one of the glass walls of combustion chamber was replaced with a steel plate to mount the pressure transducers. Hence the change in frequency can be attributed to the change in heat loss and acoustic resistance of the combustion chamber walls. The lack of other frequencies in the spectrum of flame surface area indicates that other flow features inside the chamber is not having a big impact on the heat release rate. These evidences further prove that the combustion instability is dominated by a Helmholtz-type oscillation.

The information of flame surface length inside a local region can also be used to calculate flame surface 
density $\left(\rho_{\mathrm{fs}}\right)$, which represents the average flame length inside a volume (or a region in $2 \mathrm{D}$ images) by:

$$
\rho_{\mathrm{fs}}=\lim _{\Delta x \rightarrow 0} \frac{\sum_{\Delta A} l_{\mathrm{fs}}}{(\Delta x)^{2}}
$$

where $\Delta x$ is the length of the region (bin size) and $\Delta A$ is the area of the region (bin area). The importance of flame density is that it not only indicates where the flame wrinkling is most intense, it is also believed to be directly related to local volumetric heat release rate.

From Eq.(4) we can see that the calculated flame density should be independent of bin size, and only rely on the physical processes that it is describing. However, the bin size will have effects on the results if extreme values are picked. If the bin size is too small, close to that of the pixel size, then the result would be impaired by noises generated by the acquisition system. Meanwhile, if the bin size is too large, then spacial resolution would be lost unnecessarily. Here bin size has been changed systematically until the magnitude and distribution of flame surface density is insensitive to the bin size. Figure 7 shows the calculated flame surface density of the current case with a bin size of 10 pixels $\times 10$ pixels $(394 \mu \mathrm{m} \times 394 \mu \mathrm{m})$.

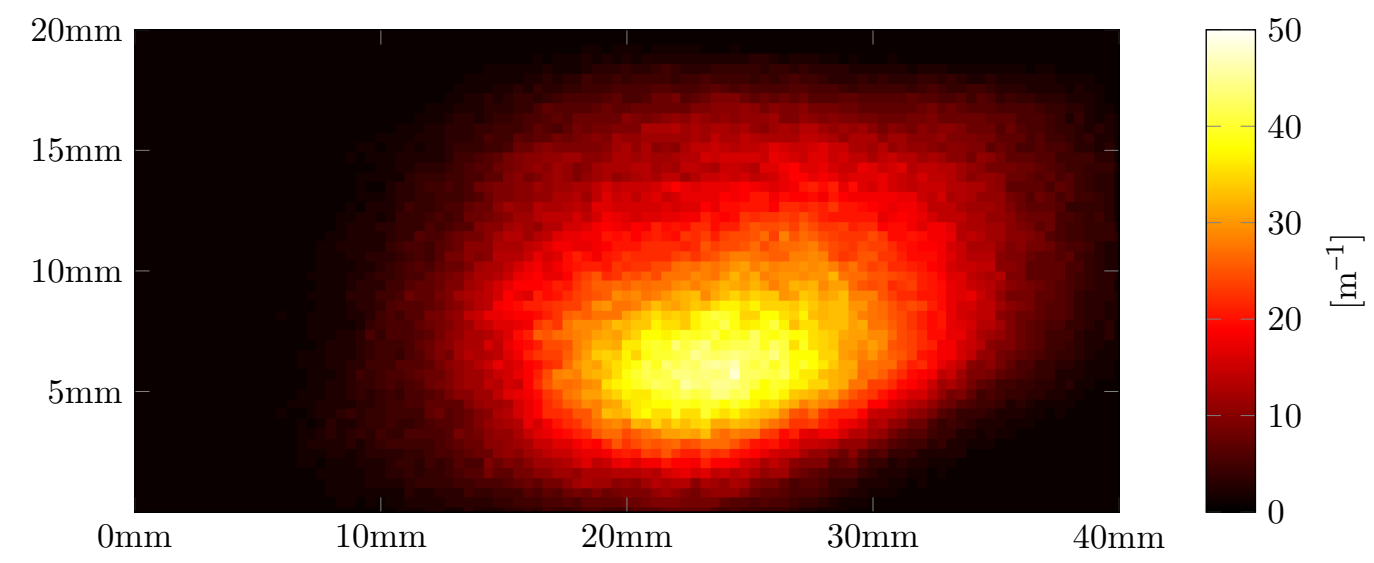

Figure 7. Flame surface density of present case

Compared to previous studies ${ }^{19}$ this result provides much higher spacial resolution and gradient resolution, while preserving most of the fundamental information. We can see that in this flame, the highest flame surface density, and hence the region where the flame is statistically most intense, is right above the surface of the flame, with a lift-off height of about $5 \mathrm{~mm}$. It is relatively flat and concentrated. These observations will aid our understanding of the driving mechanism of the combustion instability and provide key parameters in our modeling approach.

\section{Reduced Order Model}

To better understand its physical nature, we proposed a reduced order model to describe the system instability observed in our experiment. We will first derive the governing equations of the model, and then apply this model to the GTMC and compare the predictions with experimental data.

\section{A. Model description}

From the above discussions, we have concluded that the combustion instability presently observed is dominated by a Helmholtz mode. Hence our proposed reduced order model has to be based on a Helmholtz resonator analysis. Similar analysis have also been carried out previously on several different setups. ${ }^{11,14}$

Firstly, the GMTC is simplified and takes the form of an abstract system consisting of four connected bodies, as shown in figure 8. Here the four major elements of the system correspond to (0) plenum, (1) injector/swirler, (2) combustion chamber, and (3) chimney. Each element is characterized by its length and cross-section area. We assume that all gas properties (pressure, temperature, etc.) are uniform within each element. Cold mixture flows from plenum through injector into the combustion chamber. It is then ignited 
Unburnt Mixture Burnt Mixture

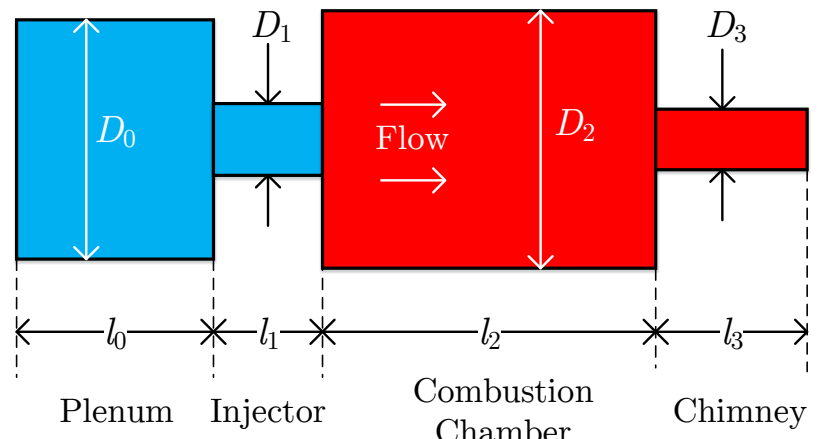

Figure 8. Schematic of the present reduced order model

and exits from the chimney. The complex flow field across the injector is neglected. Therefore, the system can be considered to be composed of four zero dimensional components.

Two of the larger elements of the system, plenum and combustion chamber, are assumed to be Helmholtz resonators. A simple Helmholtz resonator behaves like a forced oscillator, and we can write the governing equation for the internal gauge pressure $(P)$ in the form:

$$
\frac{d^{2} P}{d t^{2}}+2 \zeta \cdot \omega_{\text {res }} \cdot \frac{d P}{d t}+\omega_{\text {res }}^{2} \cdot P=f_{\text {ext }},
$$

where $\omega_{\text {res }}$ is the system resonance frequency and $f_{\text {ext }}$ is the driving term. We can also write this equation for both the plenum and combustion chamber pressure fluctuations. Specifically, the driving term for the plenum is related to the combustion chamber pressure fluctuation $\left(P_{2}^{\prime}\right)$. The driving term for the combustion chamber would be related to the heat release rate fluctuation, which takes the form of $(\gamma-1) / V \cdot d \dot{Q}^{\prime} / d t .^{11,23}$ Hence the plenum and combustion chamber pressure fluctuations would be governed by:

$$
\begin{aligned}
& \frac{d^{2} P_{0}^{\prime}}{d t^{2}}+2 \zeta_{0} \cdot \omega_{0} \cdot \frac{d P_{0}^{\prime}}{d t}+\omega_{0}^{2} \cdot P_{0}^{\prime}=\omega_{0}^{2} \cdot P_{2}^{\prime} \\
& \frac{d^{2} P_{2}^{\prime}}{d t^{2}}+2 \zeta_{2} \cdot \omega_{2} \cdot \frac{d P_{2}^{\prime}}{d t}+\omega_{2}^{2} \cdot P_{2}^{\prime}=\frac{\gamma_{\mathrm{b}}-1}{V_{2}} \cdot \frac{d \dot{Q}^{\prime}}{d t} .
\end{aligned}
$$

To solve Eq.(6), we need to relate the heat release rate fluctuation to pressure fluctuations. Heat release rate is related to the mass flow rate $(\dot{m})$ and lower heating value $\left(\Delta h_{\mathrm{g}}^{\circ}\right)$ of incoming fuel-air mixture by:

$$
\dot{Q}=\dot{m} \cdot \Delta h_{\mathrm{g}}^{\circ} \cdot \frac{\min (\phi, 1)}{\phi+A F R_{\mathrm{st}}} .
$$

Here we only consider a simplified case of premixed mixture where $\phi$ is constant. If we decompose the mass flow rate in Eq.(7) into mean $(\overline{\dot{m}})$ and fluctuation $\left(\dot{m}^{\prime}\right)$ by $\dot{m}=\overline{\dot{m}}+\dot{m}^{\prime}$, with $\overline{\dot{m}^{\prime}}=0$, then we have:

$$
\dot{Q}^{\prime}=\Delta h \cdot \dot{m}^{\prime}, \quad \text { with } \quad \Delta h=\Delta h_{\mathrm{g}}^{\circ} \cdot \frac{\min (\phi, 1)}{\phi+A F R_{\mathrm{st}}} .
$$

Fluctuation of mass flow rate can be related to the fluctuation of swirler exit velocity $\left(U_{1}^{\prime}\right)$ with a convection time delay $\left(\tau_{\mathrm{c}}\right)$ :

$$
\dot{Q}^{\prime}=\Delta h \cdot \rho_{\mathrm{u}} \cdot S_{1} \cdot U_{1}^{\prime}\left(t-\tau_{\mathrm{c}}\right) .
$$

Furthermore, the swirler exit velocity $U_{1}$ can be related to the pressure fluctuations in plenum and combustion chamber by: ${ }^{11}$

$$
\frac{d U_{1}^{\prime}}{d t}=-\frac{1}{\rho_{\mathrm{u}}} \frac{d P_{1}}{d x} \approx \frac{P_{0}^{\prime}-P_{2}^{\prime}}{\rho_{\mathrm{u}} \cdot l_{1}} .
$$


If we substitute $U_{1}^{\prime}$ in Eq.(9) with Eq.(10), and substitute $\dot{Q}^{\prime}$ in Eq.(6b) with Eq.(9), we would have the following governing equations:

$$
\begin{aligned}
& \frac{d^{2} P_{0}^{\prime}}{d t^{2}}+2 \zeta_{0} \cdot \omega_{0} \cdot \frac{d P_{0}^{\prime}}{d t}+\omega_{0}^{2} \cdot P_{0}^{\prime}=\omega_{0}^{2} \cdot P_{2}^{\prime} \\
& \frac{d^{2} P_{2}^{\prime}}{d t^{2}}+2 \zeta_{2} \cdot \omega_{2} \cdot \frac{d P_{2}^{\prime}}{d t}+\omega_{2}^{2} \cdot P_{2}^{\prime}=\left.\frac{\gamma_{\mathrm{b}}-1}{V_{2}} \cdot \frac{S_{1}}{l_{1}} \cdot \Delta h \cdot\left(P_{0}^{\prime}-P_{2}^{\prime}\right)\right|_{t-\tau_{\mathrm{c}}} .
\end{aligned}
$$

And here $\Delta h$ follows the definition shown in Eq.(8). Now we assume that the pressure in plenum and combustion chamber are sinusoidal:

$$
P_{0}^{\prime}=\widehat{P_{0}^{\prime}} \cdot \exp (\jmath \omega t), \quad P_{2}^{\prime}=\widehat{P_{2}^{\prime}} \cdot \exp \left(\jmath\left(\omega t-\psi_{20}\right)\right),
$$

where $\psi_{20}$ is the phase angle between $P_{0}^{\prime}$ and $P_{2}^{\prime}$. Substitute Eq.(12) into Eq.(11), we would obtain:

$$
\begin{aligned}
& -\omega^{2}+2 \zeta_{0} \cdot \omega_{0} \cdot \jmath \omega+\omega_{0}^{2}=\frac{\omega_{0}^{2}}{\Pi}, \\
& -\omega^{2}+2 \zeta_{2} \cdot \omega_{2} \cdot \jmath \omega+\omega_{2}^{2}=\frac{\gamma_{\mathrm{b}}-1}{V_{2}} \cdot \frac{S_{1}}{l_{1}} \cdot \Delta h \cdot \exp \left(-\jmath \omega_{\mathrm{r}} \tau_{\mathrm{c}}\right) \cdot(\Pi-1),
\end{aligned}
$$

where

$$
\Pi=\frac{P_{0}^{\prime}}{P_{2}^{\prime}}=\frac{\widehat{P_{0}^{\prime}}}{\widehat{P_{2}^{\prime}}} \cdot \exp \left(\gamma \psi_{20}\right) .
$$

In Eq.(13) the only unknowns are the system instability frequency $\omega$ and complex pressure ratio $\Pi$, all other parameters can be estimated from the configuration that this model is applied to. Hence mathematical closure is achieved.

\section{B. Comparison to present study}

We now apply the aforementioned model to our current configuration. The geometry and flow parameters of the GMTC that we used are provided in table 4.

Table 4. Model parameters used in this calculation

\begin{tabular}{ccccc}
\hline Component & Plenum & Injector & Chamber & Chimney \\
Subscript & 0 & 1 & 2 & 3 \\
\hline \hline Length $(l)[\mathrm{cm}]$ & 6.5 & 3.6 & 11.0 & 5.0 \\
Diameter $(D)[\mathrm{cm}]$ & 7.90 & $2.37^{*}$ & $8.50^{+}$ & 4.00 \\
Cross-section Area $(S)\left[\mathrm{cm}^{2}\right]$ & 49.0 & 4.4 & 72.3 & 12.6 \\
Volume $(V)\left[\mathrm{cm}^{3}\right]$ & 319 & 16 & 795 & 63 \\
Temperature $(T)[\mathrm{K}]$ & 294 & 294 & 2000 & 2000 \\
Ratio of heat capacities $(\gamma)$ & 1.4 & 1.4 & 1.3 & 1.3 \\
Speed of sound $(C)[\mathrm{m} / \mathrm{s}]$ & 344 & 344 & 864 & 864 \\
\hline
\end{tabular}

${ }^{*} D_{1}$ is calculated as the equivalent diameter combining both the inner and outer swirler.

${ }^{+}$The combustion chamber has a square cross section and $D_{2}$ is the length of its edge.

In addition to these geometry and flow parameters, we also need to calculate or estimate a series of other parameters before we can solve Eq.(13). The first of these variables are the Helmholtz resonance frequencies of plenum and combustion chamber. Both frequencies can be calculated from classical definitions of Helmholtz resonance frequencies: ${ }^{11}$

$$
\omega_{0}=\sqrt{\frac{C_{\mathrm{u}}^{2} S_{1}}{V_{0} l_{1}}}=2129 \mathrm{rad} / \mathrm{s} \approx 339 \mathrm{~Hz},
$$




$$
\omega_{2}=\sqrt{\frac{C_{\mathrm{u}}^{2} S_{1}}{V_{2} l_{1}}+\frac{C_{\mathrm{b}}^{2} S_{3}}{V_{2} l_{3}}}=5048 \mathrm{rad} / \mathrm{s} \approx 803 \mathrm{~Hz} .
$$

Notice that here the end correction to the characteristic length of the neck in typical Helmholtz analysis is not applied. Next, we need to estimate the damping ratios $\zeta_{0}$ and $\zeta_{2}$. If we only consider the effects of acoustic radiation loss, then the damping ratio of a simple Helmholtz resonator can be estimated by: ${ }^{24}$

$$
\zeta_{0}=\frac{\omega_{0} V_{0}}{4 \pi C_{\mathrm{u}}^{3}} \cdot \omega^{2} .
$$

An order-of-magnitude analysis reveals that the value of $\zeta_{0}$ is in the range of $10^{-3}$, so is $\zeta_{2}$. It can be expected that at such small values, the precise value of damping ratios will have less effect on the system instability frequency magnitude. In present calculation $\zeta_{2}$ takes the same form as $\zeta_{0}$.

The lower heating value is chosen to be $\Delta h^{\circ}=27.6 \mathrm{MJ} / \mathrm{kg} .{ }^{25}$ The only parameter left to be estimated is the convection time scale. From figure 7 we can see that the average flame lift-off height is $5 \mathrm{~mm}$ and the horizontal displacement is about $10 \mathrm{~mm}$. The injector exit velocity is estimated from reference ${ }^{2}$ to be about $12 \mathrm{~m} / \mathrm{s}$. This result in a convection time scale $\tau_{\mathrm{c}} \approx 1 \mathrm{~ms}$.

With the above parameters, Eq.(13) can be solved and yield:

$$
\begin{gathered}
\omega=2138 \mathrm{rad} / \mathrm{s} \approx 340 \mathrm{~Hz}, \\
\psi_{20} \approx 80^{\circ} .
\end{gathered}
$$

Compare these predictions with our experimental results, we can see that both the predicted system instability frequency $\omega(340 \mathrm{~Hz})$ and phase difference $\psi_{20}\left(80^{\circ}\right)$ are close to the corresponding measured values $\left(330 \mathrm{~Hz}\right.$ in section III.A or $314 \mathrm{~Hz}$ in section III.B and $50^{\circ}$ respectively). This shows the capability of present model to capture important physical processes in the system. Specifically, the system instability frequency is very close to the Helmholtz resonance frequency of plenum-injector $\left(\omega_{0}, 339 \mathrm{~Hz}\right)$. This indicates that the system is dominated by the Helmholtz-type instability of plenum, driven by the pressure fluctuation in the combustion chamber.

Currently the model has simple estimation schemes for parameters, for example the additional heat loss occurred when the combustion chamber wall is changed can not be considered. Calculation has also only been conducted at a fixed operating condition in accordance with the experimental condition. Future work is still needed to include more robust parameter estimation schemes. The effects of varying different parameters, such as equivalence ratio, mass flow rate, and fuel type, on the resulting instability frequency and phase differences need to be explored as well.

\section{Summary and Conclusions}

Multi-point pressure measurements of the combustion chamber were made on an acoustically unstable gas turbine model combustor (GTMC) operating with Dimethyl Ether (DME) to determine its primary instability mode. Time resolving simultaneous measurements of pressure and formaldehyde PLIF was then carried out at $4 \mathrm{kHz}$ to characterize the heat release rate in the combustion chamber with high temporal and spacial resolution. A reduced order model was proposed to describe the instability in the current system. Predictions of instability frequency and phase differences were compared with our experimental observations.

From this study we concluded that: $i$ ) the system is dominated by a Helmholtz type resonance, and the volume of interest is most likely the combination of plenum and injector; ii) the instability mode in the combustion chamber is of Helmholtz type, and is affected by heat loss and acoustic damping of the chimney. No vortex shedding and longitudinal standing wave effects have been observed; iii) the proposed reduced order model captures the essence of the combustion instability by accurately predicting the instability frequency and the phase difference between the plenum and the combustion chamber. Future work is needed to enhance the model robustness and explore the effects of varying mass flow rate, equivalence ratio, and fuel type on the combustion instability.

\section{Acknowledgements}

This research was funded by ONR under grant N00014-10-10561 and by the DOE-UTSR program under grant FE0007060. We thank Dr. Wolfgang Meier at DLR Stuttgart for permission to use the GTMC design. 


\section{References}

${ }^{1}$ Rayleigh, J. W. S. and Lindsay, R., The Theory of Sound, Dover Publications, 1945.

${ }^{2}$ Weigand, P., Meier, W., Duan, X. R., Stricker, W., and Aigner, M., "Investigations of swirl flames in a gas turbine model combustor I. Flow field, structures, temperature, and species distributions," Combustion and Flame, Vol. 144, No. 1-2, Jan. 2006, pp. 205-224.

${ }^{3}$ Duan, X. R., Meier, W., Weigand, P., and Lehmann, B., "Phase-resolved laser Raman scattering and laser Doppler velocimetry applied to periodic instabilities in a gas turbine model combustor," Applied Physics B, Vol. 80, No. 3, Jan. 2005, pp. 389-396.

${ }^{4}$ Meier, W., Duan, X. R., and Weigand, P., "Investigations of swirl flames in a gas turbine model combustor II. Turbulence-chemistry interactions," Combustion and Flame, Vol. 144, No. 1-2, Jan. 2006, pp. 225-236.

${ }^{5}$ Boxx, I., Arndt, C. M., Carter, C. D., and Meier, W., "High-speed laser diagnostics for the study of flame dynamics in a lean premixed gas turbine model combustor," Experiments in Fluids, Vol. 52, No. 3, Dec. 2010, pp. 555-567.

${ }^{6}$ Stöhr, M., Arndt, C. M., and Meier, W., "Effects of Damköhler number on vortex-flame interaction in a gas turbine model combustor," Proceedings of the Combustion Institute, Vol. 34, No. 2, Jan. 2013, pp. 3107-3115.

${ }^{7}$ Steinberg, A. M., Boxx, I., Stöhr, M., Carter, C. D., and Meier, W., "Flow-flame interactions causing acoustically coupled heat release fluctuations in a thermo-acoustically unstable gas turbine model combustor," Combustion and Flame, Vol. 157, No. 12, Dec. 2010, pp. 2250-2266.

${ }^{8}$ Steinberg, A. M., Boxx, I., Stöhr, M., Meier, W., and Carter, C. D., "Effects of Flow Structure Dynamics on Thermoacoustic Instabilities in Swirl-Stabilized Combustion," AIAA Journal, Vol. 50, No. 4, April 2012, pp. 952-967.

${ }^{9}$ Allison, P. M., Driscoll, J. F., and Ihme, M., "Acoustic characterization of a partially-premixed gas turbine model combustor: Syngas and hydrocarbon fuel comparisons," Proceedings of the Combustion Institute, Vol. 34, No. 2, Jan. 2013, pp. 3145-3153.

${ }^{10}$ See, Y. C. and Ihme, M., "Large eddy simulation of a partially-premixed gas turbine model combustor," Proceedings of the Combustion Institute, Sept. 2014.

${ }^{11}$ Hathout, J. P., Fleifil, M., Annaswamy, A. M., and Ghoniem, A. F., "Combustion Instability Active Control Using Periodic Fuel Injection," Journal of Propulsion and Power, Vol. 18, No. 2, March 2002, pp. 390-399.

${ }^{12}$ Candel, S., Durox, D., Schuller, T., Bourgouin, J.-F., and Moeck, J. P., "Dynamics of Swirling Flames," Annual Review of Fluid Mechanics, Vol. 46, No. 1, Jan. 2014, pp. 147-173.

${ }^{13}$ Noiray, N., Durox, D., Schuller, T., and Candel, S., "A unified framework for nonlinear combustion instability analysis based on the flame describing function," Journal of Fluid Mechanics, Vol. 615, Nov. 2008, pp. 139-167.

${ }^{14}$ Schuller, T., Durox, D., Palies, P., and Candel, S., "Acoustic decoupling of longitudinal modes in generic combustion systems," Combustion and Flame, Vol. 159, No. 5, May 2012, pp. 1921-1931.

${ }^{15}$ Palies, P., Durox, D., Schuller, T., and Candel, S., "Nonlinear combustion instability analysis based on the flame describing function applied to turbulent premixed swirling flames," Combustion and Flame, Vol. 158, No. 10, Oct. 2011, pp. $1980-1991$.

${ }^{16}$ Silva, C. F., Nicoud, F., Schuller, T., Durox, D., and Candel, S., "Combining a Helmholtz solver with the flame describing function to assess combustion instability in a premixed swirled combustor," Combustion and Flame, Vol. 160, No. 9, Sept. 2013, pp. $1743-1754$.

${ }^{17}$ Caux-Brisebois, V., Steinberg, A. M., Arndt, C. M., and Meier, W., "Thermo-acoustic velocity coupling in a swirl stabilized gas turbine model combustor," Combustion and Flame, Vol. 161, No. 12, Dec. 2014, pp. 3166-3180.

${ }^{18}$ Allison, P. M., Experimental Characterization of Combustion Instabilities and Flow-Flame Dynamics in a PartiallyPremixed Gas Turbine Model Combustor, Ph.D. thesis, University of Michigan, 2013.

${ }^{19}$ Allison, P. M., Chen, Y. T., and Driscoll, J. F., "Investigation of Dimethyl Ether Combustion Instabilities in a Partially - Premixed Gas Turbine Model Combustor Using High-Speed Laser Diagnostics," 52nd AIAA Aerospace Sciences Meeting, American Institute of Aeronautics and Astronautics, Reston, Virginia, Jan. 2014.

${ }^{20} \mathrm{Yu}, \mathrm{K} . \mathrm{H}$., Trouvé, A., and Daily, J. W., "Low-frequency pressure oscillations in a model ramjet combustor," Journal of Fluid Mechanics, Vol. 232, April 1991, pp. 47-72.

${ }^{21}$ Ahlborn, B., Seto, M. L., and Noack, B. R., "On drag, Strouhal number and vortex-street structure," Fluid Dynamics Research, Vol. 30, No. 6, June 2002, pp. 379-399.

${ }^{22}$ Allison, P. M., Chen, Y. T., Ihme, M., and Driscoll, J. F., "Coupling of flame geometry and combustion instabilities based on kilohertz formaldehyde PLIF measurements," Proceedings of the Combustion Institute, July 2014.

${ }^{23}$ Ducruix, S., Schuller, T., Durox, D., and Candel, S., "Combustion Dynamics and Instabilities: Elementary Coupling and Driving Mechanisms," Journal of Propulsion and Power, Vol. 19, No. 5, Sept. 2003, pp. 722-734.

${ }^{24}$ Kinsler, L. E., Frey, A. R., Coppens, A. B., and Sanders, J. V., Fundamentals of Acoustics, Wiley, 2000.

${ }^{25}$ Arcoumanis, C., Bae, C., Crookes, R., and Kinoshita, E., "The potential of di-methyl ether (DME) as an alternative fuel for compression-ignition engines: A review," Fuel, Vol. 87, No. 7, June 2008, pp. 1014-1030. 